\title{
Effect of Interfacial Condition between Film and Earthed Electrode on Space Charge Measurement
}

\author{
Yoshinobu Murakami, ${ }^{*}$ Tomohiro Kawashima, Naohiro Hozumi, \\ Masayuki Nagao, and Masumi Fukuma ${ }^{1}$ \\ Toyohashi University of Technology, 1-1 Hibarigaoka, Tempaku-cho, Toyohashi, Aichi 441-8580, Japan \\ ${ }^{1}$ National Institute of Technology, Matsue College, 14-4 Nishiikuma-cho, Matsue, Shimane 690-8518, Japan
}

(Received December 6, 2016; accepted April 24, 2017)

Keywords: space charge, pulsed electroacoustic method, apparent acoustic impedance, PET, interfacial condition

One electrical insulation evaluation technology is the pulsed electroacoustic method, which detects acoustic pressure due to the induced charge at the interface between the film and the electrode, and the space charge in the bulk. To improve its reliability, a measurement system for the apparent acoustic impedance of the film is introduced into the space charge measurement system. The apparent acoustic impedance of a polyethylene terephthalate (PET) film increased as the applied mechanical pressure increased. The position of the signal around the earth (lower) electrode shifted under a low mechanical pressure of $21.21 \mathrm{kN} / \mathrm{m}^{2}$, and the signal peak intensity around the earth electrode decreased at $600 \mathrm{~s}$ after the voltage application. On the other hand, the peak position shift and the decrease in peak intensity on the signal around the earth electrode were not observed under the high mechanical pressure of $1707 \mathrm{kN} / \mathrm{m}^{2}$. In the case of low mechanical pressure application, a large silicone oil layer between the PET and the earth electrode led to a charge shift due to interfacial polarization and a small peak intensity of the pressure wave generated around the interface.

\section{Introduction}

To reduce power transmission costs, power apparatuses, which are often composed of polymeric insulating materials, are being downsized. Consequently, their designed electric stress is approaching the intrinsic electric breakdown stress. Space charge is an important factor that contributes to electrical properties, especially under a dc field, including breakdown strength. To understand the space charge behavior, space charge measurements have been performed under various conditions. ${ }^{(1,2)}$

One space charge evaluation technology is the pulsed electroacoustic (PEA) method, which detects acoustic pressure due to the charge in the bulk or the induced charge at the interface between the film and the electrode. ${ }^{(3)}$ Because the acoustic properties of the entire system must be understood to improve the precision of the PEA method, a space charge measurement system equipped with a measurement function for the acoustic properties has been developed. ${ }^{(4)}$ In this study, to investigate the effect of the interfacial condition between the earth electrode (EE) and the

"Corresponding author: e-mail: murakami@ee.tut.ac.jp

http://dx.doi.org/10.18494/SAM.2017.1543 
film on the space charge measurement, this system was used to measure the acoustic impedance of the film from the EE side, and the space charge distribution affected by the interfacial condition between the $\mathrm{EE}$ and the film was investigated.

\section{Materials and Methods}

\subsection{Experimental setup}

Figure 1 schematically depicts the space charge measurement system equipped with a measurement function for the acoustic properties. ${ }^{(4)}$ The sample was an approximately $0.1-\mathrm{mm}$ thick polyethylene terephthalate (PET) film. After the EE surface was coated with silicone oil (SO) with a weight of $20 \mathrm{mg}$ and a viscosity of $1000 \mathrm{~mm}^{2} / \mathrm{s}$ (cSt), the PET film, the high-voltage semiconducting electrode (HV-SE), and the high-voltage metal electrode (HV-ME) were stacked as shown in Fig. 1. The electrode system was subjected to a specific mechanical pressure by a spring mounted in the metal high-voltage electrode.

\subsection{Estimation of interface condition between EE and PET film under specific mechanical pressure}

During an acoustic property measurement, a $5 \mathrm{~V}_{0 \mathrm{p}}$ pulse voltage generated from a pulse generator 2 (PG2) was applied to the piezoelectric device through the diode $\mathrm{D}_{1}$ and the capacitance $\mathrm{C}_{1}$. Thus, the acoustic wave propagated in the EE toward the opposite side. The same piezoelectric device received the reflection wave from the interface between the film and the EE. The output signal including the acoustic properties at the interface, which was converted into an electric signal from a pressure wave by the piezo device, traveled to a digital scope through $\mathrm{C}_{1}$, the resistance $\mathrm{R}_{1}$, and an amplifier. To estimate the interfacial condition, the reflection waveform was observed at ambient temperature before and after the space charge measurement using a homogeneous electrode system. The reflection waveform with the EE and air system was also observed as a control.

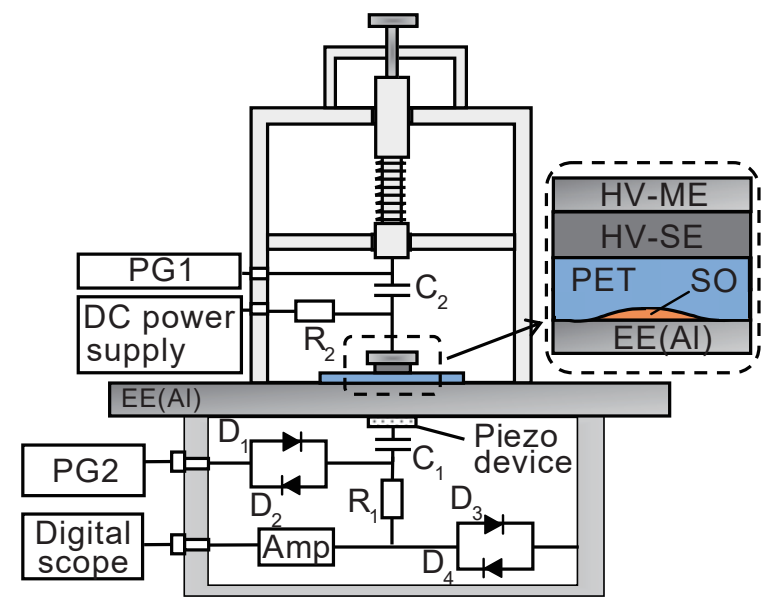

Fig. 1. (Color online) Schematic of space charge measurement system equipped with a measurement function for acoustic properties. 


\subsection{Space charge measurement}

During the space charge measurement, the output signal of the space charge also traveled to the scope through $C_{1}, R_{1}$, and the amplifier shown in Fig. 1. For the space charge measurement, the space charge distribution was observed at 1 and $600 \mathrm{~s}$ after the dc voltage application of $2 \mathrm{kV}$ at ambient temperature.

\section{Results and Discussion}

Figures 2(a) and 2(b) show typical reflection waveforms from the air/EE and PET/EE interfaces under the pressure of about $1707 \mathrm{kN} / \mathrm{m}^{2}$, respectively. The apparent acoustic impedance of the film was employed in the estimation of the interfacial condition between the film and the EE. The apparent acoustic impedance of the PET film, $Z_{P E T}\left[\mathrm{~kg} / \mathrm{m}^{2} / \mathrm{s}\right]$, was estimated using

$$
Z_{P E T}=\frac{P_{r(\text { Air } / E E)}-P_{r(\text { Material } / E E)}}{P_{r(\text { Air } / E E)}+P_{r(\text { Material } / E E)}} Z_{E E}
$$

where $P_{r(A i r / E E)}$ is the magnitude between the first and second peaks in the reflection waveform from the air/EE interface, $\operatorname{Pr(Material/EE)}$ is the magnitude between the first and second peaks in the reflection waveform from a certain material at the EE/EE interface, and $Z_{E E}$ is the acoustic impedance of $\mathrm{Al}\left(=17.51 \times 10^{6} \mathrm{~kg} / \mathrm{m}^{2} / \mathrm{s}\right) .^{(5)}$

Figures 3(a) and 3(b) show the apparent acoustic impedance before the voltage application as a function of mechanical pressure and the apparent acoustic impedance before or after the voltage application, respectively. The symbols and error bars denote the average of five samples and their standard deviation, respectively. The acoustic impedance below $21.21 \mathrm{kN} / \mathrm{m}^{2}$ pressure shown in Fig. 3(a) agreed with that of the silicone oil only measured using this system. Additionally, the acoustic impedance increased with the increase in pressure, and approached that of the PET film $\left(Z_{P E T}\right)$, which is estimated from the values of the measured velocity and density. ${ }^{(6,7)}$ As shown in Fig. 3(b), the acoustic impedance under $21.21 \mathrm{kN} / \mathrm{m}^{2}$ pressure after the voltage application is higher than that before the voltage application. The contact area ratio of the PET film to the EE, which has a higher

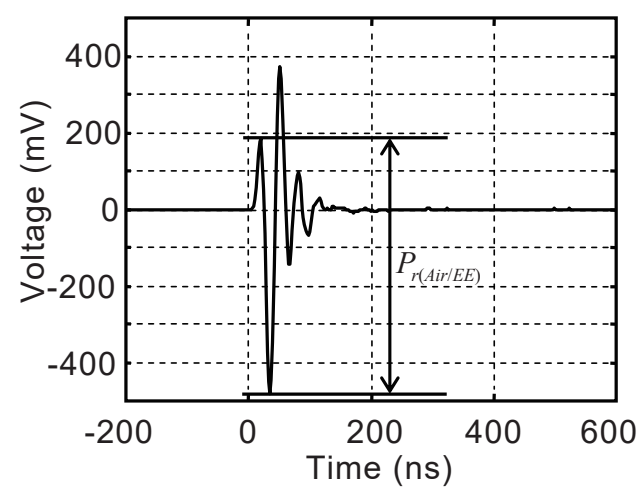

(a)

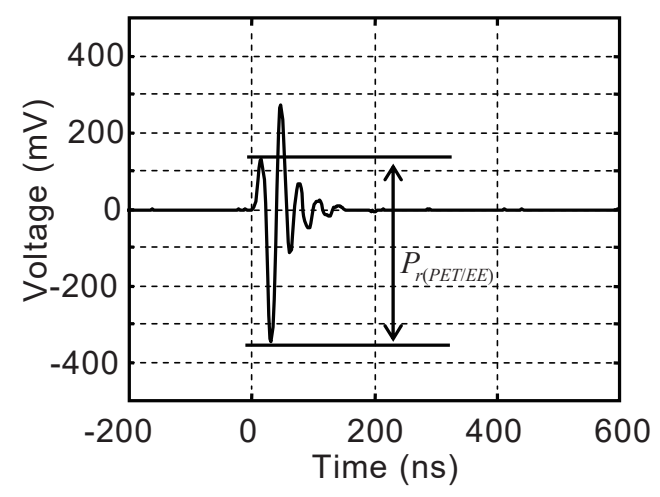

(b)

Fig. 2. Typical reflection waveforms from (a) air/EE interface and (b) PET/EE interface under a pressure of about $1707 \mathrm{kN} / \mathrm{m}^{2}$. 


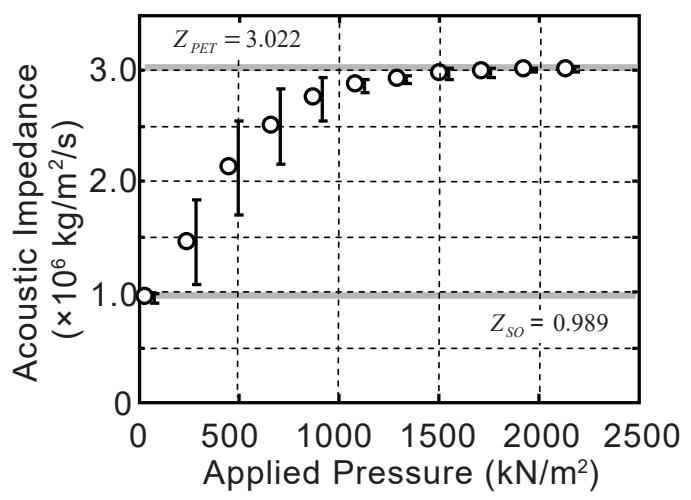

(a)

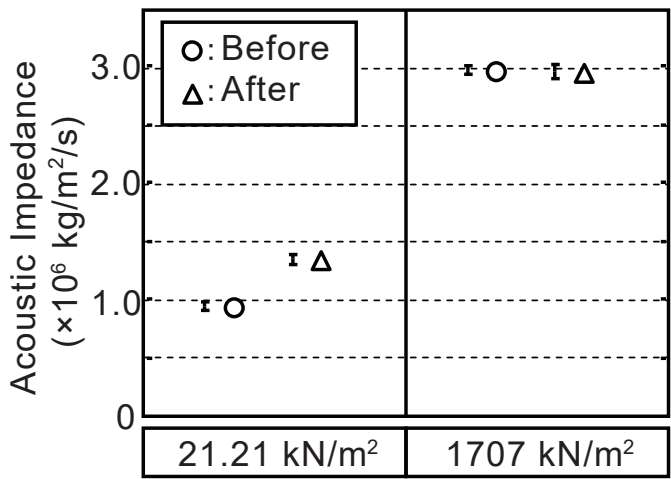

(b)

Fig. 3. Apparent acoustic impedances as a function of mechanical pressure (a) before voltage application and (b) before or after voltage application.

acoustic impedance $Z_{P E T}$ than the SO $\left(Z_{S O}\right)$, increased when the SO was removed from the PET/EE interface with the passage of time under low pressure application.

Figure 4 shows the original signal before the deconvolution analysis and the space charge distribution under different pressures. The original signal obtained $1 \mathrm{~s}$ after the voltage application under each pressure was used as the reference in the deconvolution analysis. The zero (0) in the horizontal axis indicates the cathode peak position of the original signal or the space charge distribution obtained $1 \mathrm{~s}$ after the voltage application. As shown in Figs. 4(a) and 4(b), the cathode peak intensity of the original signal and the space charge distribution under a low pressure decreased and the cathode peak position shifted to the anode side, suggesting that the negative charge traveled to the silicone oil layer to induce interfacial polarization or the negative space charge was injected into the bulk. On the other hand, the signals under a high pressure did not change over time as shown in Figs. 4(c) and 4(d).

Figure 5 schematically shows the contact condition around the EE. When the attenuation of the pressure wave is neglected, the acoustic wave $P_{P E T / E E}$ propagating to the piezo device, which is generated at the complete interface between the PET film and the EE, is expressed as

$$
P_{P E T / E E}=\frac{Z_{E E}}{Z_{P E T}+Z_{E E}} \sigma(0) e_{p(P E T)}(t) \approx 0.850 \sigma(0) e_{p(P E T)}(t),
$$

where $\sigma(0)\left(\mathrm{C} / \mathrm{m}^{2}\right)$ is the charge density at the PET/EE interface and $e_{p(P E T)}(t)(\mathrm{V} / \mathrm{m})$ is the pulse field applied to the PET film. On the other hand, when the silicon oil layer is present between the PET film and the EE, the acoustic waves $P_{S O / E E}$ and $P_{P E T / S O}$ before and after the interface polarization, propagating to the piezo device, are expressed as

$$
\begin{aligned}
P_{S O / E E} & =\frac{Z_{E E}}{Z_{S O}+P_{E E}} \sigma(0)\left(\frac{\varepsilon_{r(S O)} d_{P E T}}{\varepsilon_{r(S O)} d_{P E T}-d_{S O}\left(\varepsilon_{r(S O)}-\varepsilon_{r(P E T)}\right)} \frac{\varepsilon_{r(P E T)}}{\varepsilon_{r(S O)}}\right) e_{p(P E T)}(t), \\
& \approx 0.587 \sigma(0) e_{p(P E T)}(t),
\end{aligned}
$$




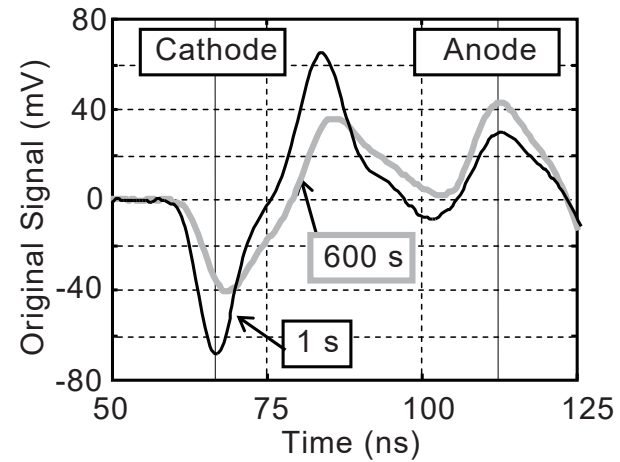

(a)

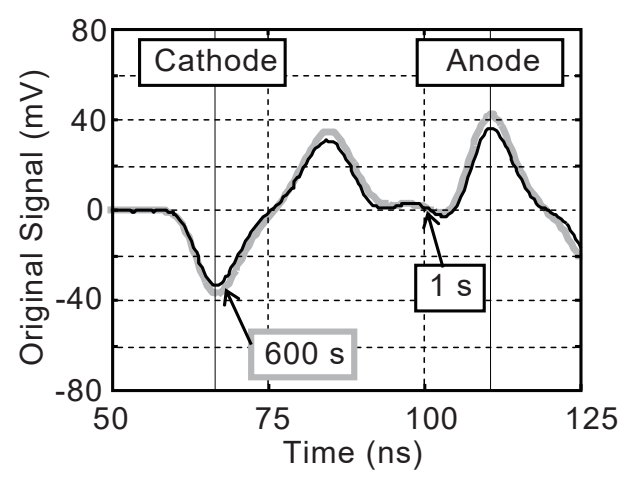

(c)

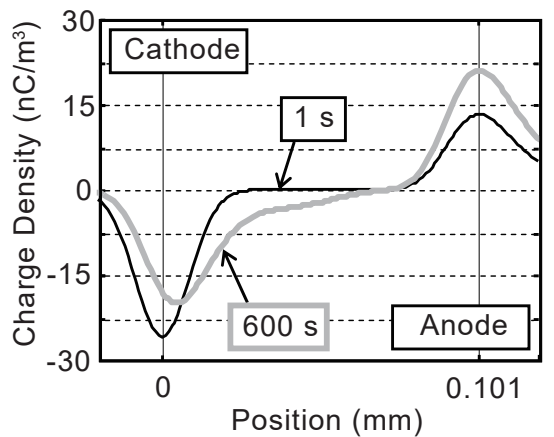

(b)

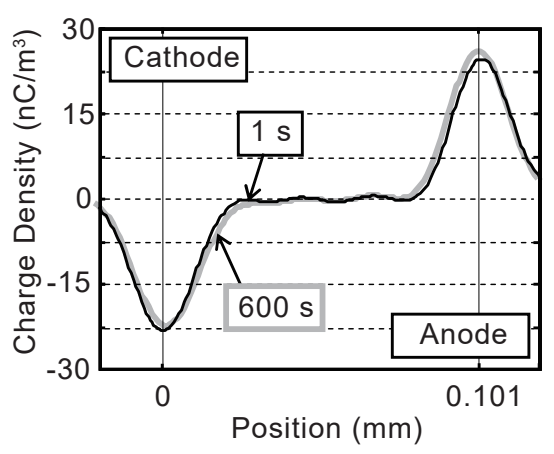

(d)

Fig. 4. Original signals under applied pressures of (a) 21.21 and (c) $1707 \mathrm{kN} / \mathrm{m}^{2}$, and charge densities under applied pressures of (b) 21.21 and (d) $1707 \mathrm{kN} / \mathrm{m}^{2}$.

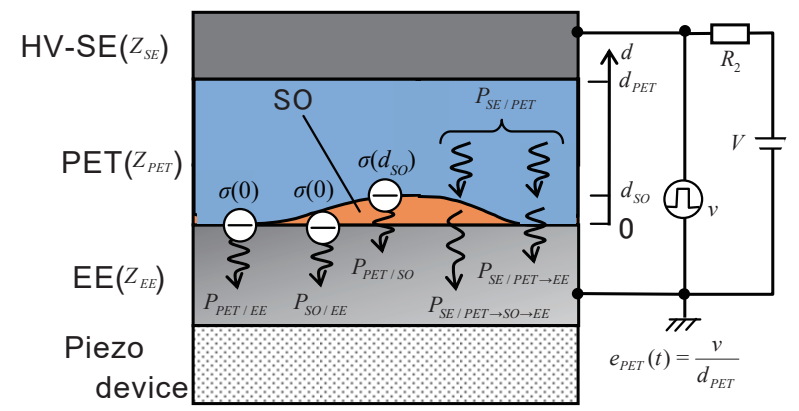

Fig. 5. (Color online) Schematic of the contact condition between the PET film and the lower electrode.

$$
\begin{aligned}
P_{P E T / S O} & =\frac{2 Z_{E E}}{Z_{S O}+Z_{E E}} \frac{Z_{S O}}{Z_{S O}+Z_{E E}} \sigma\left(d_{S O}\right)\left(\frac{\varepsilon_{r(S O)} d_{P E T}}{\varepsilon_{r(S O)} d_{P E T}-d_{S O}\left(\varepsilon_{r(S O)}-\varepsilon_{r(P E T)}\right)} \frac{\varepsilon_{r(P E T)}}{\varepsilon_{r(S O)}}\right) e_{p(P E T)}(t), \\
& \approx 0.275 \sigma(0) e_{p(P E T)}(t),
\end{aligned}
$$

where $\sigma\left(d_{S O}\right)[=\sigma(0)]\left(\mathrm{C} / \mathrm{m}^{2}\right)$ is the charge density at the PET/SO interface, $d_{P E T}(=0.2 \mathrm{~mm})$ is the thickness of the PET film, $d_{S O}(=0.0025 \mathrm{~mm})$ is the thickness of the SO layer, $\varepsilon_{r(P E T)}\left(=3.0^{(8)}\right)$ is the permittivity of the PET film, and $\varepsilon_{r(S O)}\left(=2.76^{(9)}\right)$ is the permittivity of SO. 
$P_{P E T / S O}$ after the interface polarization (600 s after voltage application) is smaller than $P_{S O / E E}$ before the interface polarization ( $1 \mathrm{~s}$ after voltage application), as well as the results, as shown in Figs. 4(a) and 4(b). Similarly, the equations of the acoustic waves $P_{S E / P E T \rightarrow P E T \rightarrow E E}$ and $P_{S E / P E T \rightarrow P E T \rightarrow S O \rightarrow E E}$, which are generated at the interface between the semiconducting electrode (SE) and the PET film, and propagate to the piezo device either not-through or through the silicone oil layer, are respectively expressed as

$$
\begin{gathered}
P_{S E / P E T \rightarrow P E T \rightarrow E E}=\frac{2 Z_{E E}}{Z_{E E}+Z_{P E T}} P_{S E / P E T} \approx 1.71 P_{S E / P E T}, \\
P_{S E / P E T \rightarrow P E T \rightarrow S O \rightarrow E E}=\frac{2 Z_{E E}}{Z_{E E}+Z_{P E T}} \frac{2 Z_{S O}}{Z_{S O}+Z_{P E T}} P_{S E / P E T} \approx 0.84 P_{S E / P E T},
\end{gathered}
$$

where $P_{S E / P E T}$ is the acoustic wave generated at the SE/PET interface, which propagates to the interface between the PET film and the EE (or PET and SO). The magnitude relationships of these equations are also in agreement with the results obtained 1 and $600 \mathrm{~s}$ after voltage application as shown in Figs. 4(a) and 4(b).

In Fig. 4, the negative space charge under $21.21 \mathrm{kN} / \mathrm{m}^{2}$ pressure seems to be injected into the PET film. Owing to the low field of about $20 \mathrm{kV} / \mathrm{mm}$, the accumulation of the space charge in the PET film is difficult under these experimental conditions. Furthermore, the acoustic impedances below a pressure of $1707 \mathrm{kN} / \mathrm{m}^{2}$ are almost the same before and after the voltage application. Therefore, these space charge distributions may be the apparent space charge distributions, which include the effect of the adhesion degree between the PET film and the EE.

\section{Conclusions}

To contribute to a highly precise measurement by the PEA method, the space charge distribution and acoustic impedance of the film were measured using a space charge measurement system equipped with the measurement function for the acoustic properties. As the mechanical pressure decreases (or when the contact condition between the PET film and the EE is poor), the negative space charge accumulates in the bulk. The applied field and the observation that the acoustic impedances of the PET film before and after the voltage application agree when the pressure is sufficient suggest that the apparent space charge occurs when PET has weak contact with the EE.

\section{References}

1 T. Doi, Y. Tanaka, and T. Takada: Annu. Rep. Conf. Electr. Insul. Dielectr. Phenom. (IEEE, 1989) p. 722.

2 Y. Murakami, M. Nemoto, S. Okuzumi, S. Masuda, M. Nagao, N. Hozumi, and Y. Sekiguchi: IEEE Trans. Dielectr. Electr. Insul. 15 (2011) p. 33.

3 T. Maeno and K. Fukunaga: IEEE Trans. Dielectr. Electr. Insul. 3 (1996) 754.

4 M. Fukuma, R. Funo, Y. Murakami, M. Nagao, and N. Hozumi: Proc. 2011 Int. Symp. Electr. Insul. Mater. CD-ROM (IEEJ, 2011) p. 722.

5 Y. Murakami, T. Sugiyama, T. Kawashima, M. Nagao, and M. Fukuma: Proc. 11th Int. Conf. Proper. Appl. Dielectr. Mater. (IEEE, 2015) No. SC2-1.

6 J. Rochab and V. R. Singh: Indian J. Pure Appl. Phys. 42 (2004) p. 595.

7 National Institute of Technology and Evaluation: Human Chemical Database (2007) p. 2 (in Japanese). 
8 Y. Sakurauti: Plasttic Gijyutsu Dokuhon (Kogyo Chosakai Publishing Co., Ltd., Tokyo, 1987) p. 18 (in Japanese).

9 Shin-etsu Chem. Corp.: Technical Report (2003) p. 3 (in Japanese).

\section{About the Authors}

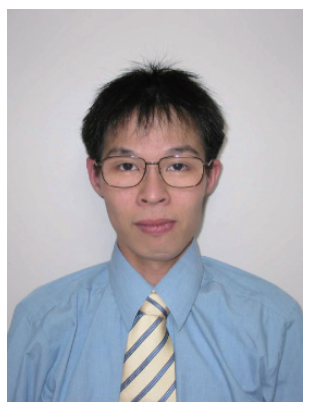

Yoshinobu Murakami received his B.S., M.S., and Ph.D. degrees from Toyohashi University of Technology, Japan, in 1997, 1999, and 2002, respectively. From 2001 to 2003, he was an assistant professor at Nagano College of Technology, Japan. He was an assistant professor from 2003 to 2008 and a lecturer from 2008 to 2010 at Toyohashi University of Technology, Japan. Since 2010, he has been an associate professor at Toyohashi University of Technology. His research interests are in the development of measurement and the diagnostic systems for dielectric and electrical insulation phenomena, and the development of a functional composite insulating material.

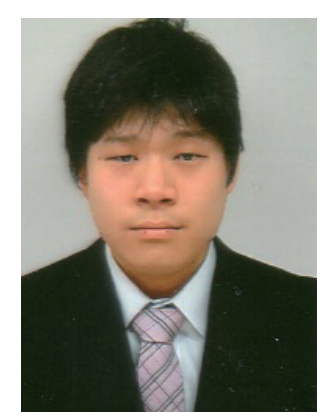

Tomohiro Kawashima received his B.S. and M.S. degrees from Toyohashi University of Technology, Japan, in 2011 and 2013, respectively. Since 2013, he has been an assistant professor at Toyohasi Univesity of Technology, Japan. His research interest is in cryogenic insulation.

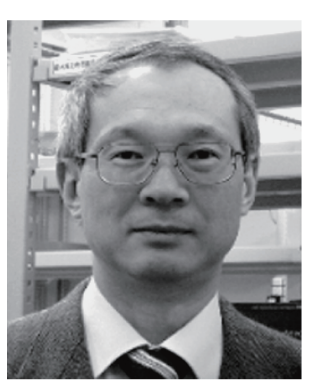

Naohiro Hozumi received his B.S. and M.S. degrees from Waseda University, Japan, in 1981 and 1983, respectively. From 1983 to 1999, he joined the Central Research Institute of Electric Power Industry, Japan. He was an associate professor from 1999 to 2006 at Toyohashi University of Technology and a professor from 2006 to 2011 at Aichi Institute of Technology, Japan. Since 2011, he has been a professor at Toyohashi University of Technology. His research interests are in insulating materials and diagnosis for high-voltage equipment and measurement and signal processing for medical devices.

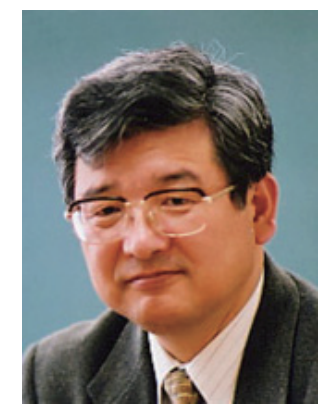

Masayuki Nagao received his B.S., M.S., and Ph.D. degrees from Nagoya University, Japan, in 1973, 1975, and 1978, respectively. From 1978 to 1980, he was a lecturer at Nagoya University, Japan. He was an associate professor from 1985 to 1999 and a professor from 1999 to 2016 at Toyohashi University of Technology. Since 2016, he has been a project professor at Toyohashi University of Technology. His research interests are in the high-field electrical properties of polymers and insulation degradation phenomena. 


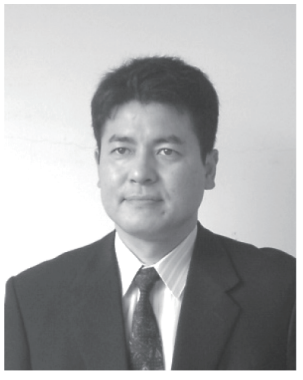

Masumi Fukuma received his B.S. and M.S. degrees from Toyohashi University of Technology, Japan, in 1983 and 1985, respectively. From 1985 to 1991, he joined Sumitomo Electric Industries, Ltd., Japan. He was a lecturer from 1991 to 1994 and an associate professor from 1994 to 2002 at Matsue College of Technology, Japan. Since 2002, he has been a professor at Matsue College of Technology. His research interests are in space charge measurement systems and electric double-layer capacitors. 